\title{
Compensatory Neural Activity in Response to Cognitive Fatigue
}

\author{
Chao Wang, ${ }^{1}$ Amy Trongnetrpunya, ${ }^{1}$ Immanuel Babu Henry Samuel, ${ }^{1}$ Mingzhou Ding, ${ }^{1}$ and Benzi M. Kluger ${ }^{2,3}$ \\ ${ }^{1} \mathrm{~J}$. Crayton Pruitt Family Department of Biomedical Engineering, University of Florida, Gainesville, Florida 32611 and Departments of ${ }^{2}$ Neurology and \\ ${ }^{3}$ Psychiatry, University of Colorado Denver, Aurora, Colorado 80045
}

Prolonged continuous performance of a cognitively demanding task induces cognitive fatigue and is associated with a time-related deterioration of objective performance, the degree of which is referred to cognitive fatigability. Although the neural underpinnings of cognitive fatigue are poorly understood, prior studies report changes in neural activity consistent with deterioration of task-related networks over time. While compensatory brain activity is reported to maintain motor task performance in the face of motor fatigue and cognitive performance in the face of other stressors (e.g., aging) and structural changes, there are no studies to date demonstrating compensatory activity for cognitive fatigue. High-density electroencephalography was recorded from human subjects during a 160 min continuous performance of a cognitive control task. While most time-varying neural activity showed a linear decline over time, we identified an evoked potential over the anterior frontal region which demonstrated an inverted U-shaped time-on-task profile. This evoked brain activity peaked between 60 and $100 \mathrm{~min}$ into the task and was positively associated with better behavioral performance only during this interval. Following the peak and during subsequent decline of this anterior frontal activity, the rate of performance decline also accelerated. These findings demonstrate that this anterior frontal brain activity, which is not part of the primary task-related activity at baseline, is recruited to compensate for fatigue-induced impairments in the primary task-related network, and that this compensation terminates as cognitive fatigue further progresses. These findings may be relevant to understanding individual differences in cognitive fatigability and developing interventions for clinical conditions afflicted by fatigue.

Key words: cognitive; event related potential; fatigue; Stroop

\section{Significance Statement}

Fatigue refers to changes in objective performance and subjective effort induced by continuous task performance. We examined the neural underpinnings of cognitive fatigue in humans using a prolonged continuous performance task and high-density electroencephalography with the goal of determining whether compensatory processes exist to maintain performance in the face of fatigue. We identified brain activity demonstrating an inverted U-shaped time-on-task profile. This brain activity showed features consistent with a compensatory role including: peaking between 60 and 100 min into the task, a positive association with behavioral performance only during this interval, and accelerated performance decline following its peak. These findings may be relevant to understanding individual differences in cognitive fatigue and developing interventions for clinical conditions afflicted by fatigue.

\section{Introduction}

Fatigue may be defined in terms of behavioral, physiological, and psychological changes induced by prolonged task performance

\footnotetext{
Received Sept. 17, 2015; revised Feb. 1, 2016; accepted Feb. 29, 2016.

Author contributions: B.M.K. designed research; C.W. and I.B.H.S. performed research; C.W., A.T., I.B.H.S., and M.D. analyzed data; C.W., A.T., I.B.H.S., M.D., and B.M.K. wrote the paper.

This work was supported by the U.S. Army Research Laboratory and the U. S. Army Research Office (Contract/ Grant W911NF-10-1-0192), the Colorado Clinical and Translational Sciences Institute KL2 Program (NIH/National Center for Advancing Translational Sciences Grant 8 KL2 TR000156-05), the National Institutes of Health (NIA Grant R21 AG044862; NINDS Grant 5 K02 NS080885-02), and the National Science Foundation (Grant BCS-1439188).

The authors declare no competing financial interests.

Correspondence should be addressed to Dr. Benzi M. Kluger, Department of Neurology, University of Colorado Denver, Mail Stop B-185, 12631 East 17th Avenue, Aurora, C0 80045. E-mail: benzi.kluger@ucdenver.edu.
}

(Kluger et al., 2013). Whereas the behavioral and psychological aspects of fatigue are well described, less is known regarding physiological changes underlying fatigue. A major challenge facing researchers is determining the significance of time-varying physiological signals that may reflect (1) functional deterioration of systems underlying task performance, (2) engagement of systems associated with monitoring effort/fatigue, (3) learning or other time-dependent processes not associated with fatigue, and/or (4) engagement of compensatory processes to maintain 
performance. Prior neurophysiologic studies of prolonged cognitive tasks report changes consistent with functional deterioration of task-related networks, enhanced effort, and increased fatigue perception (Gevins et al., 1987; Ishii et al., 2013; Esposito et al., 2014; Tanaka et al., 2014). To our knowledge, there have been no studies demonstrating engagement of compensatory brain activity to maintain performance for cognitively fatiguing tasks. This is a significant gap, as understanding compensatory mechanisms may help us predict individual variability of cognitive fatigability, understand how neurologic lesions cause fatigue, and develop therapeutic interventions. As compensatory brain activity is involved in maintaining motor performance in the face of fatigue and cognitive performance in the face of sleep deprivation (Drummond et al., 2000), environmental noise (Wong et al., 2009), and aging (Barulli and Stern, 2013), we hypothesized that similar compensatory mechanisms are also engaged with cognitive fatigability.

To test this hypothesis, we recorded brain activity using high-density electroencephalography (EEG) during a $160 \mathrm{~min}$ continuous cognitive control task (cued Stroop task) in healthy volunteers to identify temporal and functional profiles of neural activity consistent with compensation. We examined changes in preparatory activity time locked to the color-naming instructional cue because prior studies showed proactive cognitive control is more susceptible to fatigue than other cognitive functions (Lorist et al., 2005). Taking a completely data driven approach, we first examined variability in global field power (GFP) over the course of the 160 min task to identify time periods of interest relative to cue onset (Lehmann and Skrandies, 1980). We then used event-related potentials (ERPs) to identify topographic regions of interest. Finally, we modeled changes of ERP amplitudes over time in relation to behavioral performance to deduce its underlying functional role.

\section{Materials and Methods}

The experimental protocol was approved by the University of Florida Institutional Review Board. Sixteen college students free from neurological disorders and with normal or corrected-to-normal vision provided written informed consent and participated in the study in exchange for course credit. All participants were right-handed and native English speakers. They were asked to refrain from consuming caffeine or nicotine on the day of testing. All experiments began at 9:00 A.M. Data from 14 subjects $(18-33$ years of age, $19.5 \pm 3.9$ (mean $\pm \mathrm{SD}$ ) years, seven females, seven males) were included in the analyses reported here. Two participants were excluded for (1) excessive motion artifacts and (2) poor electrode-scalp connection.

\section{Study design and experimental paradigm}

Participants sat in an acoustically and electrically shielded room and performed a computerized cued Stroop task (Cohen et al., 1999) continuously for $160 \mathrm{~min}$ (Wang et al., 2014). Instructions and stimuli were displayed visually on a PC. Each trial began with an instructional cue ("word" or "color") presented for $1 \mathrm{~s}$, followed by a word stimulus ("red," "blue," or "green") written in colored letters for a random cuetarget interval of 1, 3, or $5 \mathrm{~s}$ in duration. For the "word" task, participants were instructed to read the word, whereas for the "color" task, participants were instructed to name the color of the letters. Sixty percent of the trials were congruent (e.g., the word "red" written in red letters) and $40 \%$ were incongruent (e.g., the word "red" written in green letters). Reaction times (RTs) were determined by voice activation software, and verbal responses were manually recorded. After a brief practice session (30 trials), participants were fitted with EEG electrodes and asked to perform the task for a single $160 \mathrm{~min}$ session. Breaks for any purpose were taken only if requested and resulted in the termination of the experiment and the exclusion of the participant.

\section{EEG recording and preprocessing}

The EEG data were recorded using a 128 channel BioSemi Active Two System (BioSemi) at a sampling rate of $1024 \mathrm{~Hz}$. A left central posterior electrode served as reference. The three-dimensional coordinates of each electrode as well as three fiducial landmarks were determined by means of a Polhemus spatial digitizer. Off-line data preprocessing was performed using BESA 5.3 (BESA), EEGLAB (Delorme and Makeig, 2004), and custom scripts. The continuous EEG data from each subject were first visually inspected. Data segments contaminated by large head movement artifacts were rejected. Channels with poor signal quality were rejected and replaced by spatially interpolated data. The EEG signals were then bandpass filtered between 0.1 and $83 \mathrm{~Hz}$ and downsampled to 250 $\mathrm{Hz}$. The data within the period from -200 to $1500 \mathrm{~ms}$ relative to cue onset $(0 \mathrm{~ms})$ were epoched for analysis. Artifacts, including eye movements and eye blinks, temporal muscle activity, and line noise, were removed from data epochs using the Infomax independent component analysis (ICA) algorithm implemented in EEGLAB (Jung et al., 2000). After ICA, any epoch with incorrect behavioral response and voltage exceeding $75 \mu \mathrm{V}$ in any scalp channel was rejected from further analysis. The total rejection rate of trials from each subject was between $\sim 7$ and $\sim 30 \%$. The remaining artifact-free signals were rereferenced to the average reference. Since the exact electrode locations are slightly different from subject to subject, spherical spline interpolation was applied to project each subject's 128 channels of data into a standard 10-10 montage with 81 channels (Anderson and Ding, 2011). All of the following analyses were performed on the standardized 81 channel data.

\section{ERP analysis and time-on-task effects}

We focused on color-naming trials. Compared to word-reading trials, color-naming trials required increased cognitive control and were thus more prone to the deleterious effects of cognitive fatigue. ERPs evoked by the color cue were thus expected to provide a more sensitive measure of the time-on-task effect.

ERP averaging for different time blocks. To assess the time-on-task effect on ERPs, we segmented the $160 \mathrm{~min}$ data into 40 min time blocks. A 40 min time block contained $\sim 100$ color-naming trials for each subject after preprocessing, sufficient for obtaining a reliable estimate of the ERPs. However, if we segmented the $160 \mathrm{~min}$ data into $40 \mathrm{~min}$ blocks without overlap, it would result in only four time blocks $(0-40,40-80,80-120$, and $120-160 \mathrm{~min}$ ) for analysis. To increase the temporal resolution, we applied a $40 \mathrm{~min}$ moving window through the $160 \mathrm{~min}$ data with a $10 \mathrm{~min}$ moving step. This resulted in 13 overlapped time blocks (e.g., $0-40$, $10-50, \ldots, 120-160 \mathrm{~min}$ ) for analysis. Compared to the nonoverlapped 4 time blocks, the overlapped 13 time blocks can reveal the time-on-task effect with better temporal resolution. For each time block, the ERP waveforms for each scalp electrode were extracted by averaging preprocessed epochs of color-naming trials separately for each subject. The extracted ERP waveforms were low-pass filtered with the cutoff set at $30 \mathrm{~Hz}$. A $200 \mathrm{~ms}$ period preceding the cue onset was used as baseline.

Global field power. GFP is a measure of global strength of evoked responses and provides an index of the overall level of brain activation as a function of time (Lehmann and Skrandies, 1980; Murray et al., 2008). Mathematically, GFP at each time point is defined as the SD of the electric potentials from all electrodes with respect to the average reference at that time point. GFP time course helps to identify the time periods in which brain activities were mostly affected by time on task.

The GFP functions were first computed for each color-cue epoch (from -200 to $1500 \mathrm{~ms}$ relative to cue onset) and then averaged across all the color-cue trials in each 40 min time block. GFP changes over time blocks were then tested via a one-way repeated measures ANOVA using a $100 \mathrm{~ms}$ moving window. The time periods with $p$ values of $<0.1$ were selected as times of interest (TOIs) for further ERP analyses.

Analysis of spatial distribution of time-on-task effects. To study the spatial distribution of time-on-task effect on ERPs, we tested the ERP changes over time blocks for each scalp electrode. The mean amplitudes of ERP within each TOI identified by the GFP analysis were first computed for each electrode for each time-on-task block. The changes of ERP amplitudes over different time-on-task blocks were then tested via one-way repeated measures ANOVA. Tested $p$ values were adjusted for 
A

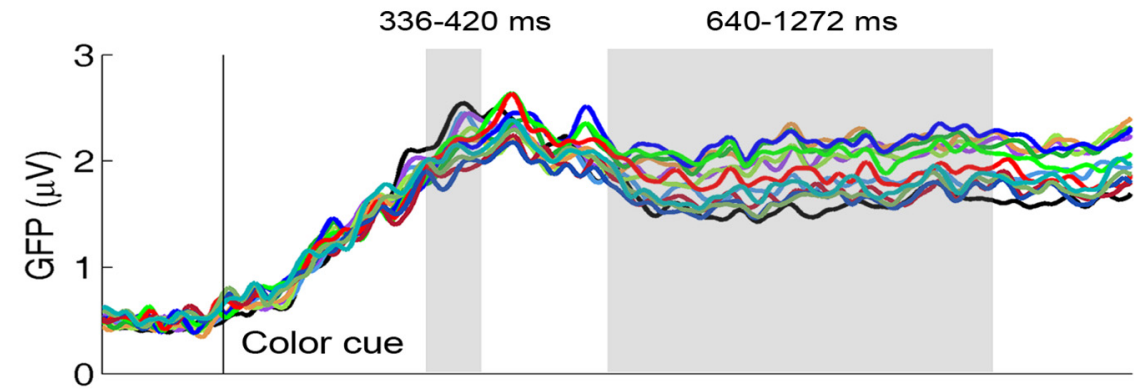

B
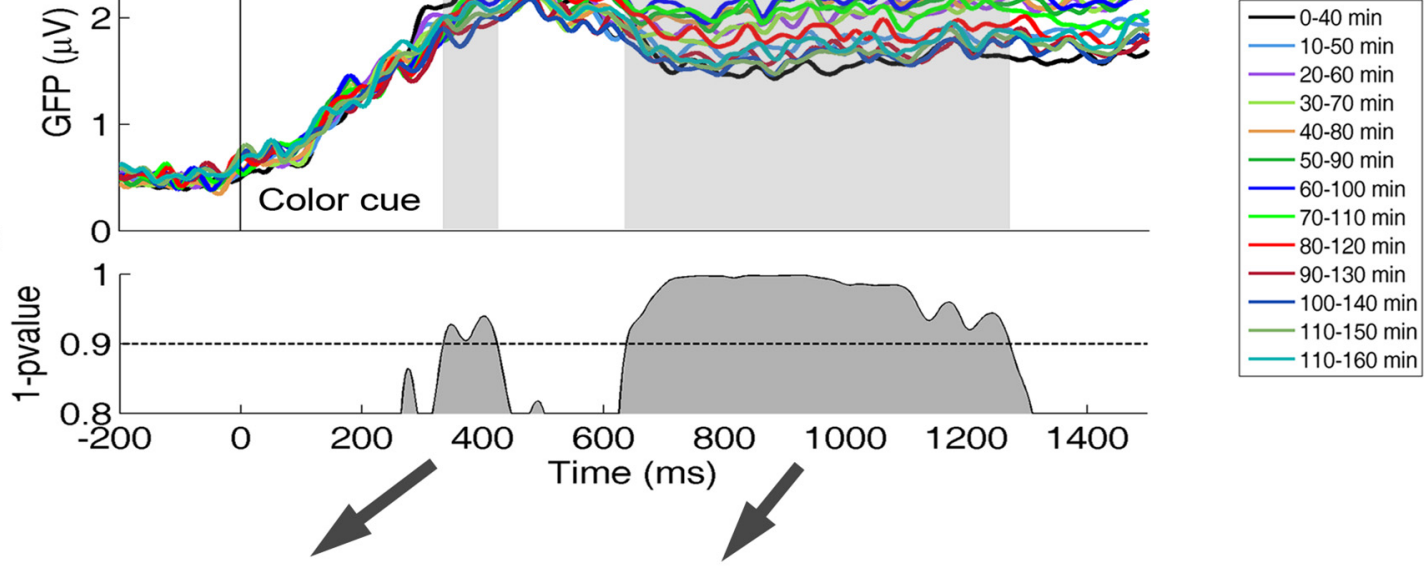

C

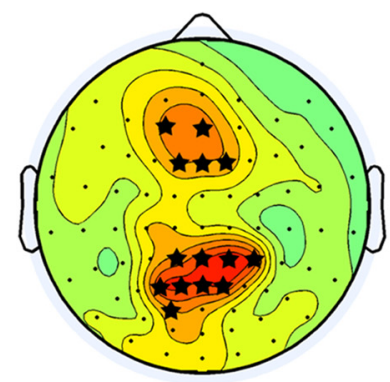

$640-1272 \mathrm{~ms}$

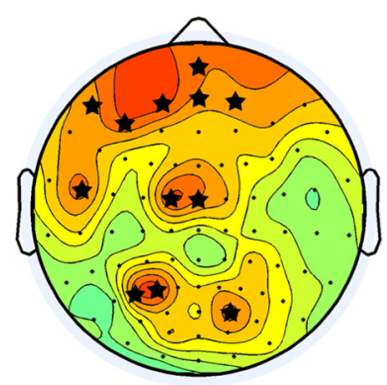

D

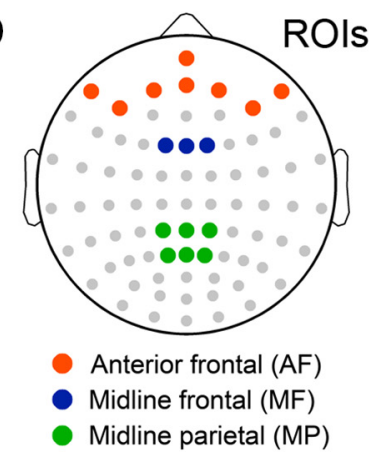

Figure 1. Time-on-task effects on evoked activities and their spatial distributions. $A$, The color cue-related GFP waveforms for different time-on-task blocks. Time 0 on the $x$-axis denotes cue onset. $\boldsymbol{B}$, Results of statistical tests on changes of GFP waveforms over time blocks. The dash line indicates $p=0.1$. Evoked periods with $p<0.1$ are shaded in Figure $2 A$ and selected for further analyses. C, Statistical topographical maps of ERP changes over time blocks for the period of 336-420 ms and the period of $640-1272 \mathrm{~ms}$. The color scales denote $F$ values from ANOVA tests. Electrodes with significant ERP changes ( $p<0.05$, corrected) are marked by asterisks. $D$, ROls selected for further analyses. The electrode layout is the standard $10-10$ system. The anterior frontal $\mathrm{ROI}$ contains electrodes at AF9, AF7, Fp1, Fpz, Nz, Fp2, AF8, and AF10. The midline frontal ROI contains electrodes at F1, Fz, and F2. The midline parietal ROI contains electrodes at $C P 1$, $C P z$, $C P 2$, $P 1$, $\mathrm{PZ}$, and P2.

multiple comparisons by the number of electrodes using the HolmBonferroni correction (Holm, 1979). The adjusted $p$ values $<0.05$ were considered as statistically significant. Regions with at least three contiguous significant electrodes were selected as regions of interest (ROIs) for further analyses.

Analysis of temporal dynamics of time-on-task effects. Page's trend tests (Page, 1963) were applied to test whether the ERP amplitudes from the ROIs consistently declined over time-on-task blocks across subjects. The $p$ values were obtained from 10,000 within-subject permutations of timeon-task blocks. The temporal trajectories of mean ERP amplitudes averaged across subjects were examined by linear and quadratic regressions. The differences of ERP amplitudes between two time-on-task blocks were tested via paired $t$ tests.

Relationship between ERP and RT. To ascertain the functional significance of changes in brain responses to the onset of the color-cue, we examined the relationship between ERPs and behavior by sorting each subject's trials into equal-sized fast, medium, and slow RT groups with $50 \%$ overlap. Congruent trials and incongruent trials were sorted separately and then combined. The differences of ERP waveforms for different RT groups were tested via repeated measures ANOVA within a 100 ms moving window.

\section{Results}

Performance over the 160 min task showed a significant time-ontask effect with slowing of RT consistent with cognitive fatigue (1169 $\pm 46 \mathrm{~ms}$ in first $40 \mathrm{~min}$ block to $1402 \pm 72 \mathrm{~ms}$ in last block; repeated measures ANOVA with Greenhouse-Geisser correction, $\left.F_{(1.76,22.93)}=8.07, p=0.003\right)$ and a trend toward worsening accuracy $(3.39 \pm 0.65 \%$ error rate in first block vs $5.34 \pm 1.12 \%$ in last block; $\left.F_{(1.81,23.49)}=2.93, p=0.078\right)$.

GFP waveforms are shown in Figure $1 A$. Statistical analyses (Fig. $1 B$ ) reveal that brain responses with significant time-on-task variability occurred within two time periods relative to the cue; an early period of 336-420 ms and a late period of 640-1272 ms. Figure $1 C$ shows the statistical topographical maps of time-ontask effect on ERPs during these two TOIs with the early ERP component demonstrating activity in midline frontal (MF) and parietal (MP) regions and the late ERP component involving the anterior frontal (AF) region. Based on these statistical topographical maps, we selected the MF and MP regions as ROIs for the early ERP analysis and the AF region as the ROI for the late ERP analysis (Fig. 1D).

The temporal trajectories of the amplitudes of the three ERP components over time-on-task blocks are shown in Figure 2. ANOVA confirmed that the time-on-task effects were significant for these three ERP components $\left(F_{(1,12)}=3.52, p=0.0003\right.$ for early ERP from MF; $F_{(1,12)}=5.39, p<0.0001$ for early ERP from $\mathrm{MP} ; F_{(1,12)}=4.41, p<0.0001$ for late ERP from AF). For the early ERP components from MF and MP, the amplitudes consistently 
declined over time-on-task blocks ( $p=$ 0.0002 for MF, $p<0.0001$ for MP, Page's trend test). Their temporal trajectories of the amplitude averaged across subjects followed a linear relationship with timeon-task (MF, $r^{2}=0.74, p=0.0002$; MP, $r^{2}=0.84, p<0.0001$; Fig. $\left.2 A\right)$. In contrast, for the late ERP component from $\mathrm{AF}$, the amplitude first significantly increased ( $p=0.0016$ ) from the first block $(0-40 \mathrm{~min})$ to the middle block $(60-100$ $\mathrm{min})$, and then significantly decreased $(p=0.037)$ from the middle block (60$100 \mathrm{~min})$ to the end block ( $120-160 \mathrm{~min})$. The temporal trajectories of the amplitudes averaged across subjects followed a nonlinear inverted $\mathrm{U}$-shaped relationship $\left(r^{2}=0.78, p=0.0005\right.$; Fig. $\left.2 B\right)$.

To further ascertain the functional significance of the late AF ERP, we examined the relationships between the amplitude of this ERP and RT by timeon-task blocks. At the beginning of the experiment $(0-40 \mathrm{~min})$, there was no significant correlation between the ERP and behavioral performance (Fig. $3 A$ ), suggesting this ERP had a limited functional role before fatigue. For the middle time-on-task block (60-100 min), we observed a significant association be-

tween ERP amplitude and RT, with larger ERPs being associated with faster RTs (Fig. 3B), suggesting that this ERP was recruited to maintain task performance. For the last time block (120-160 min), the previously observed ERP association with RT was diminished with significant effects now only found for a later time period relative to cue onset (1400-1500 ms; Fig. $3 C$ ). This finding parallels the ERP amplitude decrease over late time blocks, suggesting compensation is also time limited.

As a final examination of the potential compensatory role of the AF ERP, we separated the 0-160 min time-on-task interval into a compensation phase $(0-80 \mathrm{~min})$ and a decompensation phase ( $80-160 \mathrm{~min})$ based on the rise and fall of this ERP's amplitude (Fig. 4A). Since compensation would help maintain task performance, the rate of performance decline would accelerate in the decompensation phase. Figure $4 B-D$ displays three distinct task performance measures during each phase: error rate, unusually long RT trials $(>2 \mathrm{~s})$ thought to reflect momentary lapses in attention (Smith and Nutt, 1996; Smith et al., 2005), and mean RT as a function of time-on-task blocks. The $2 \mathrm{~s}$ threshold for unusually long RT trials was selected based on the $90 \%$ cutoff point of the RT distribution. As seen in Figure 4, $B$ and $C$, error rate and long RT trials were not significantly different from the beginning time block $(p>$ 0.05 , paired $t$ test) during the compensation phase. Significant increases $(p<0.05)$ of error rate and long RT trials were observed only during the decompensation phase. For mean RT, although we observed significant increases $(p<0.05)$ during both the compensation phase and the decompensation phase (Fig. $4 D$ ), the slope of RT slowing during the compensation phase was significantly smaller $(p=0.0460)$ than during the decompensation phase (Fig. $4 E$ ).

\section{Discussion}

Consistent with our initial hypothesis, we identified neural activity engaged to compensate for fatigue-related cognitive performance decline. Specifically, we identified an anterior frontal ERP that demonstrated several characteristics strongly suggestive of a compensatory role including: (1) no change in amplitude or correlation with performance during baseline/prefatigue performance, (2) increase in amplitude during compensatory period with significant positive association with task performance, (3) subsequent decompensation followed by a final period of disengagement and loss of association with task performance, and (4) accelerated performance decline during the period of disengagement. This pattern of results is consistent with our predictions of compensatory neural activity as well as prior studies of compensatory central neural activity for motor performance (Liu et al., 2002).

Although the precise neural source of this compensatory activity cannot be determined solely on the basis of our ERP data, the topography of these results suggest a source distinct from other cue-related neural activity associated with nonfatigued performance of this task (Wang et al., 2015). This ERP is also notable for demonstrating task-related changes in activity much later in the preparatory period than previously reported task-related ERPs (700-1100 vs $250-450 \mathrm{~ms})$. Our data-driven approach is critical in allowing identification of this ERP. Since it is not associated with task performance in the initial prefatigue time period, it would not have been possible to identify it based on traditional ERP paradigms or a priori theoretical considerations.

We speculate that this ERP may reflect anterior cingulate or presupplementary motor area activity; both areas have been associated with modulation of effort and cognitive control (Sohn and Lee, 2007; Vassena et al., 2014). Alternatively, this ERP may reflect anterior orbitofrontal activity, an area previously associated with subjec- 

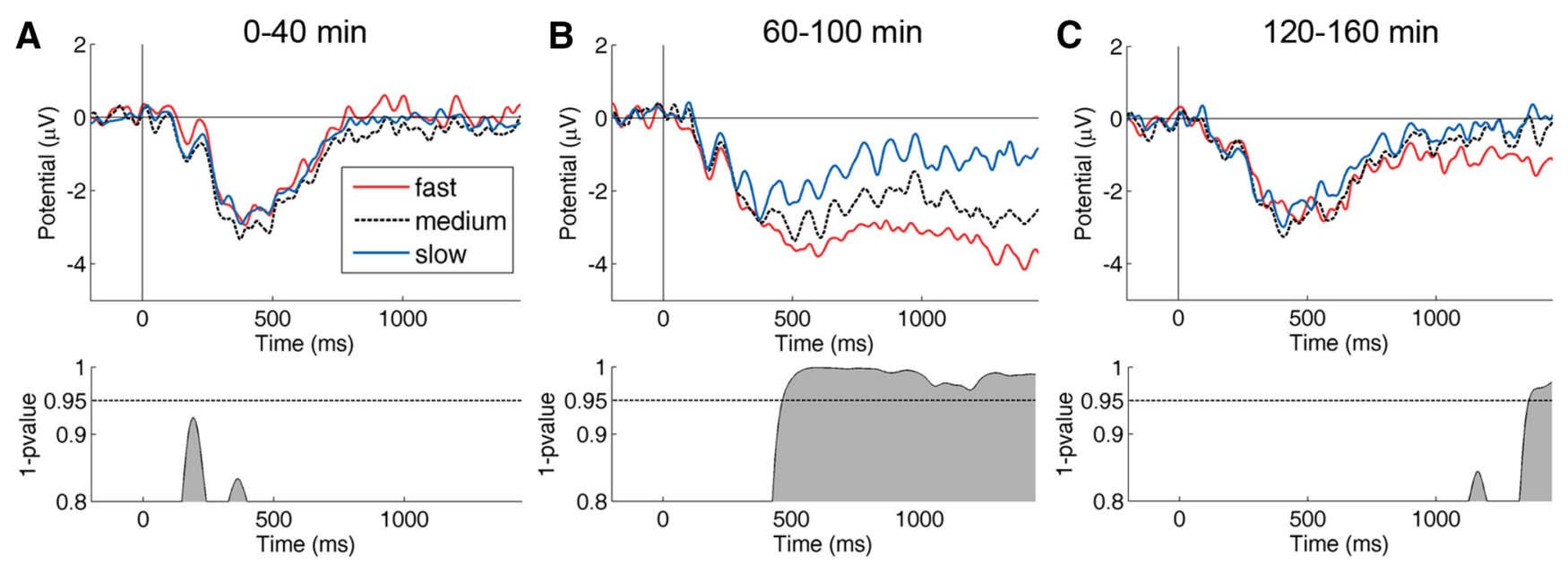

Figure 3. Anterior frontal ERP and behavior. $A-C$, ERP waveforms from the anterior frontal ROl for fast, medium, and slow RT trials during the first time block $(0-40$ min; $A)$, the middle time block $(60-100 \mathrm{~min} ; \boldsymbol{B})$, and the last time block (120-160 min; $\boldsymbol{C}$. Time 0 on the $x$-axis denotes cue onset. Results of statistical tests on the ERP differences among different RT groups are plotted at the bottom of the figure. A significance threshold of $p<0.05$ was applied. During the first time block, no significant ERP difference was observed for different RT groups. During the middle time block, significant ERP separations were found in the range of $468-1450 \mathrm{~ms}$. During the last time block, significant ERP separations were found only in the range of $1360-1450 \mathrm{~ms}$.
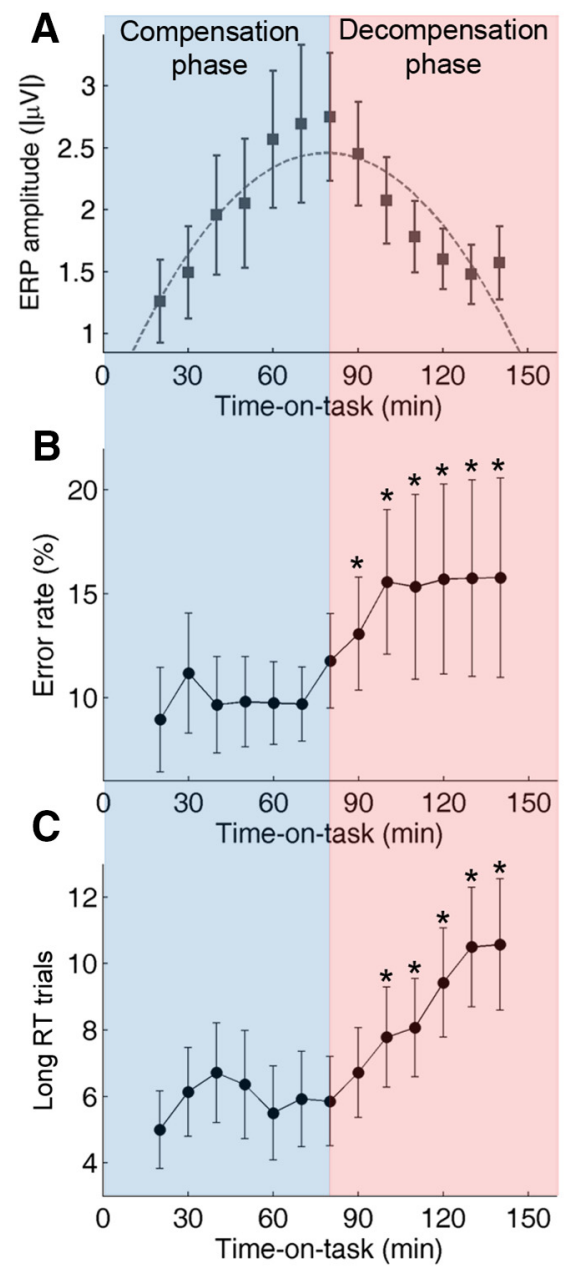
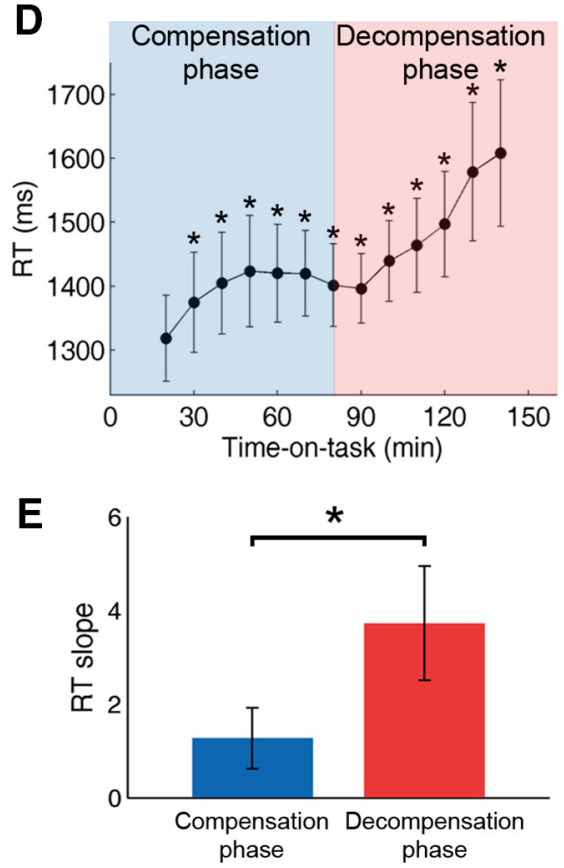

Figure 4. Task performance during compensation and decompensation. $A$, ERP amplitude during $640-1272 \mathrm{~ms}$ from the anterior frontal ROl was used to define the compensation phase $(0-80 \mathrm{~min}$, shaded in blue) and the decompensation phase (80-160 min, shaded in red). $\boldsymbol{B}$, Error rate in color incongruent condition as a function of time on task. Significant error rate increases compared to the beginning time block are marked by asterisks. C, Long RT trials (number of trials with RTs longer than $2 \mathrm{~s}$ ) in the color incongruent condition as a function of time on task. Significant increases of long RT trials compared to the beginning time block are marked by asterisks. $\boldsymbol{D}, \mathrm{RT}$ in the color incongruent condition as a function of time on task. Significant RT increases compared to the beginning time block are marked by asterisks. $E$, Slope of RT increase is higher in the decompensation phase than in the compensation phase $(p<0.05)$. Error bars indicate SE. tive sensations of fatigue in healthy subjects performing a cognitive task (Tajima et al., 2010) and in multiple sclerosis (MS) patients with chronic fatigue complaints (Pardini et al., 2013). Interestingly, activity in this area was associated with improved motor performance among MS patients in the same study, suggesting a potential compensatory role. This area has also been posited to be involved in monitoring the ongoing balance between task rewards and fatiguerelated costs (Boksem and Tops, 2008). Future studies using magnetoencephalography or functional MRI could be pursued to delineate the neuroanatomical source of the anterior frontal ERP.

Although anterior frontal activity may be associated with ocular artifacts (Hughes and Miller, 1988) and eye strain may be seen in fatiguing protocols (Filtness et al., 2014), this activity would be expected to increase linearly across the entire task period and show no association with behavioral performance. Similarly, subjective sensations of fatigue and costbenefit estimations of effort would be expected to increase across the entire time course of the task and would not be expected to be associated with task performance. Learning effect is another possible confounding factor. However, in the behavioral data, we did not observe any performance improvement from the very beginning, suggesting that learning effect is not a major concern. In addition, prior neuroimaging studies found that the principal effect of practice is a reduction in the extent and magnitude of activity in cortical networks (Kassubek et al., 2001; Chein and Schneider, 2005). In contrast, we observed an increase of anterior frontal 
brain activity over early time-on-task period. It is therefore unlikely that our findings are due to practice/learning effect.

In summary, this study demonstrates that neural mechanisms exist to maintain cognitive performance in the face of fatigue. We propose that fatigue-related compensatory activity and decompensation may prove a useful model to better understand compensatory brain activity in a relatively short time frame in healthy younger adults and may shed light on these processes in aging and neurological illness. Similarities between our findings and compensatory mechanisms reported in aging include increased recruitment, posterior to anterior shift, and prolonged/delayed recruitment (Eyler et al., 2011; Barulli and Stern, 2013; Störmer et al., 2013). Interestingly, the orbitofrontal cortex (Habeck et al., 2012; Burianová et al., 2013) and anterior cingulate (Ansado et al., 2012), possible sources of the observed anterior frontal ERP, have both been associated with beneficial compensatory responses in older adults with and without cognitive impairment.

\section{References}

Anderson KL, Ding M (2011) Attentional modulation of the somatosensory mu rhythm. Neuroscience 180:165-180. CrossRef Medline

Ansado J, Monchi O, Ennabil N, Faure S, Joanette Y (2012) Load-dependent posterior-anterior shift in aging in complex visual selective attention situations. Brain Res 1454:14-22. CrossRef Medline

Barulli D, Stern Y (2013) Efficiency, capacity, compensation, maintenance, plasticity: emerging concepts in cognitive reserve. Trends Cogn Sci 17: 502-509. CrossRef Medline

Boksem MA, Tops M (2008) Mental fatigue: costs and benefits. Brain Res Rev 59:125-139. CrossRef Medline

Burianová H, Lee Y, Grady CL, Moscovitch M (2013) Age-related dedifferentiation and compensatory changes in the functional network underlying face processing. Neurobiol Aging 34:2759-2767. CrossRef Medline

Chein JM, Schneider W (2005) Neuroimaging studies of practice-related change: fMRI and meta-analytic evidence of a domain-general control network for learning. Cogn Brain Res 25:607-623. CrossRef

Cohen JD, Barch DM, Carter C, Servan-Schreiber D (1999) Contextprocessing deficits in schizophrenia: converging evidence from three theoretically motivated cognitive tasks. J Abnorm Psychol 108:120-133. CrossRef Medline

Delorme A, Makeig S (2004) EEGLAB: an open source toolbox for analysis of single-trial EEG dynamics including independent component analysis. J Neurosci Meth 134:9-21. CrossRef

Drummond SP, Brown GG, Gillin JC, Stricker JL, Wong EC, Buxton RB (2000) Altered brain response to verbal learning following sleep deprivation. Nature 403:655-657. CrossRef Medline

Esposito F, Otto T, Zijlstra FR, Goebel R (2014) Spatially distributed effects of mental exhaustion on resting-state FMRI networks. PLoS One 9:e94222. CrossRef Medline

Eyler LT, Sherzai A, Kaup AR, Jeste DV (2011) A review of functional brain imaging correlates of successful cognitive aging. Biol Psychiatry 70: 115-122. CrossRef Medline

Filtness AJ, Anund A, Fors C, Ahlström C, Akerstedt T, Kecklund G (2014) Sleep-related eye symptoms and their potential for identifying driver sleepiness. J Sleep Res 23:568-575. CrossRef Medline

Gevins AS, Morgan NH, Bressler SL, Cutillo BA, White RM, Illes J, Greer DS, Doyle JC, Zeitlin GM (1987) Human neuroelectric patterns predict performance accuracy. Science 235:580-585. CrossRef Medline

Habeck C, Risacher S, Lee GJ, Glymour MM, Mormino E, Mukherjee S, Kim S, Nho K, DeCarli C, Saykin AJ, Crane PK (2012) Relationship between baseline brain metabolism measured using [(1)(8)F]FDG PET and memory and executive function in prodromal and early Alzheimer's disease. Brain Imaging Behav 6:568-583. CrossRef Medline
Holm S (1979) A simple sequentially rejective multiple test procedure. Scand J Stat 6:65-70.

Hughes JR, Miller JK (1988) Eye movements on brain maps. Clin Electroencephalogr 19:210-213. CrossRef Medline

Ishii A, Tanaka M, Shigihara Y, Kanai E, Funakura M, Watanabe Y (2013) Neural effects of prolonged mental fatigue: a magnetoencephalography study. Brain Res 1529:105-112. CrossRef Medline

Jung TP, Makeig S, Humphries C, Lee TW, McKeown MJ, Iragui V, Sejnowski TJ (2000) Removing electroencephalographic artifacts by blind source separation. Psychophysiology 37:163-178. Medline

Kassubek J, Schmidtke K, Kimmig H, Lücking CH, Greenlee MW (2001) Changes in cortical activation during mirror reading before and after training: an fMRI study of procedural learning. Cogn Brain Res 10: 207-217. CrossRef

Kluger BM, Krupp LB, Enoka RM (2013) Fatigue and fatigability in neurologic illnesses: proposal for a unified taxonomy. Neurology 80:409-416. CrossRef Medline

Lehmann D, Skrandies W (1980) Reference-free identification of components of checkerboard-evoked multichannel potential fields. Electroen Clin Neuro 48:609-621. CrossRef

Liu JZ, Dai TH, Sahgal V, Brown RW, Yue GH (2002) Nonlinear cortical modulation of muscle fatigue: a functional MRI study. Brain Res 957: 320-329. CrossRef Medline

Lorist MM, Boksem MA, Ridderinkhof KR (2005) Impaired cognitive control and reduced cingulate activity during mental fatigue. Brain Res Cogn Brain Res 24:199-205. CrossRef Medline

Murray MM, Brunet D, Michel CM (2008) Topographic ERP analyses: a step-by-step tutorial review. Brain Topogr 20:249-264. CrossRef Medline

Page EB (1963) Ordered hypotheses for multiple treatments: a significance test for linear ranks. J Am Stat Assoc 58:216-230. CrossRef

Pardini M, Bonzano L, Roccatagliata L, Mancardi GL, Bove M (2013) The fatigue-motor performance paradox in multiple sclerosis. Sci Rep 3:2001. Medline

Smith A, Nutt D (1996) Noradrenaline and attention lapses. Nature 380: 291. CrossRef Medline

Smith A, Sutherland D, Christopher G (2005) Effects of repeated doses of caffeine on mood and performance of alert and fatigued volunteers. J Psychopharmacol 19:620-626. CrossRef

Sohn JW, Lee D (2007) Order-dependent modulation of directional signals in the supplementary and presupplementary motor areas. J Neurosci 27: 13655-13666. CrossRef Medline

Störmer VS, Li SC, Heekeren HR, Lindenberger U (2013) Normal aging delays and compromises early multifocal visual attention during object tracking. J Cogn Neurosci 25:188-202. CrossRef Medline

Tajima S, Yamamoto S, Tanaka M, Kataoka Y, Iwase M, Yoshikawa E, Okada H, Onoe H, Tsukada H, Kuratsune H, Ouchi Y, Watanabe Y (2010) Medial orbitofrontal cortex is associated with fatigue sensation. Neurol Res Int 2010:671421. Medline

Tanaka M, Ishii A, Watanabe Y (2014) Neural effects of mental fatigue caused by continuous attention load: a magnetoencephalography study. Brain Res 1561:60-66. CrossRef Medline

Vassena E, Silvetti M, Boehler CN, Achten E, Fias W, Verguts T (2014) Overlapping neural systems represent cognitive effort and reward anticipation. PLoS One 9:e91008. CrossRef Medline

Wang C, Ding M, Kluger BM (2014) Change in intraindividual variability over time as a key metric for defining performance-based cognitive fatigability. Brain Cogn 85:251-258. CrossRef Medline

Wang C, Ding M, Kluger BM (2015) Functional roles of neural preparatory processes in a cued Stroop task revealed by linking electrophysiology with behavioral performance. PLoS One 10:e0134686. CrossRef Medline

Wong PC, Jin JX, Gunasekera GM, Abel R, Lee ER, Dhar S (2009) Aging and cortical mechanisms of speech perception in noise. Neuropsychologia 47:693-703. CrossRef Medline 